\title{
Scabies Associated with Granulomatous Dermatitis
}

\author{
Jessica Harris Rajiv Nathoo Vladimir Vincek \\ Department of Dermatology, University of Florida College of Medicine, \\ Gainesville, FL, USA
}

\section{Keywords}

Scabies · Granuloma · Granulomatous · Dermatitis

\begin{abstract}
Scabies is a relatively common parasitic infection, which has sometimes been associated with the formation of granuloma annulare. We report the case of an 86-year-old female who developed a non-necrotizing granulomatous dermatitis after a scabies infestation. This case highlights the importance of considering scabies in the differential diagnosis when granulomatous dermatitis is noted histologically that cannot be explained by other causes.
\end{abstract}

(C) 2017 The Author(s)

Published by S. Karger AG, Basel

\section{Case Report}

An 86-year-old female presented to the dermatology clinic with a 3-week history of an itchy rash located on the trunk and arms. Her past medical history included glaucoma, cataracts, and breast cancer treated with radiation therapy. She had no history of sarcoidosis, tuberculosis, granuloma annulare (GA), atopic diathesis, or new medications. Review of systems was negative for recent fevers, chills, or night sweats.

Physical examination revealed pink urticarial-like papules and plaques scattered over the trunk and extremities (Fig. 1). No burrows were identified. The clinical differential diagnosis included arthropod bites, drug eruption, urticaria, and urticarial vasculitis. 
A punch biopsy was obtained from the left posterior shoulder and showed a sarcoptic mite in association with granulomatous dermatitis (Fig. 2a). A burrow with a Sarcoptes mite was present within the stratum corneum (Fig. 2b), and non-necrotizing granulomas were present within the dermis (Fig. 2c). Granulomas were surrounded with lymphocytes and scattered eosinophils. Stains for infectious organisms (acid fast bacilli and Grocott's methenamine stain) were negative, and Alcian blue stain did not demonstrate dermal mucin.

The patient received treatment for scabies with ivermectin and symptomatic management with levocetirizine resulting in the resolution of her rash.

\section{Discussion}

Scabies is a contagious infestation of the skin caused by a mite, Sarcoptes scabiei var. hominis, which lives in the stratum corneum of the human host. Scabies has a wide spectrum of clinical manifestations and, similar to syphilis, has been described as a "great imitator" [1]. Scabies infestation is typically characterized by an intensely pruritic eruption with burrows, papules, or vesicles. However, scabies can mimic other skin conditions such as psoriasis, atopic dermatitis, diaper dermatitis, allergic contact dermatitis, and irritant contact dermatitis [2]. Diagnosis can be confirmed by recovering mites, mite fecal pellets, or eggs from a skin scraping. Dermatoscopy can aid in detecting burrows on examination. Biopsy can sometimes but not always reveal the mite, ova, or scybala in the stratum corneum. However, even if scabies is not found in the biopsy, the dermis typically demonstrates features of a hypersensitivity reaction with a perivascular lymphoid infiltrate with eosinophils. In those cases with high clinical suspicion for scabies along with the dermal findings of hypersensitivity reaction, empirical treatment is often initiated. In the case of our patient, if scabies was not found in the biopsy, the presence of only granulomatous dermatitis would have led to a different differential diagnosis.

Scabies infestations have been noted to be associated with and possibly trigger other conditions such as urticaria, lichen ruber, psoriasis, dermatitis herpetiformis, Darier's disease, Hailey-Hailey disease, Grover disease, and bullous pemphigoid [1,3]. There are a few case reports of GA, a common benign granulomatous disease of unknown etiology, being induced by scabies infection. In previously reported cases, scabies infestation was followed by generalized GA or localized GA [3-5]. One case described a patient with a 10-year history of relapsing generalized GA that became resistant to topical therapy after infection with scabies [1]. Previous reports hypothesize that scabies can trigger other diseases because of a Koebner phenomenon [5]. GA may appear following diseases such as mycobacterial and fungal infections, herpes simplex infection, herpes zoster, erythema multiforme minor, insect bites, vaccines, and lymphoma, and it sometimes occurs in the same site as the initial disease process [3].

Studies indicate that antigens from the scabies mite induce a cellular and humoral response in the host $[2,6]$. In one study, the clinical severity of the disease was reflected in differences in the type and magnitude of humoral and cellular responses, and a nonprotective T-helper 2 response occurred in patients who developed crusted scabies [6]. Immunological changes induced by scabies infestation such as elevated immunoglobulin E may act as a trigger for immune dysregulation [3]. This immune dysregulation may lead to a variety of different reactions including a non-necrotizing granulomatous dermatitis as in this case. Despite several previous reports of GA associated with scabies, our case was not GA as evidenced by the histology including the absence of dermal mucin. 
We hypothesize that scabies infestation can cause a granulomatous reaction that is either rare or overlooked and could potentially lead to a misdiagnosis if not considered. Therefore, it is important to consider scabies in the differential diagnosis of a granulomatous dermatitis that cannot otherwise be explained.

\section{Acknowledgement}

We are indebted to Dr. Michael Wangia and Dr. Christopher Spock for their assistance.

\section{Statement of Ethics}

Informed consent was obtained from the patient prior to publication.

\section{Disclosure Statement}

The authors declare no conflicts of interest.

\section{References}

1 Wilsmann-theis D, Wenzel J, Gerdsen R, Uerlich M, Bieber T: Granuloma annulare induced by scabies. Acta Derm Venereol 2003;83:318.

-2 Arlian LG, Feldmeier H, Morgan MS: The potential for a blood test for scabies. PLoS Negl Trop Dis 2015;9:e0004188.

-3 Piana S, Pizzigoni S, Tagliavini E, Serra S, Albertini G: Generalized granuloma annulare associated with scabies. Am J Dermatopathol 2010;32:518-520.

-4 Vignale RA, Casella de Vilaboa E, Paciel J, Bruno J: Disseminated annular granuloma in a patient with scabies. Med Cutan Ibero Lat Am 1990;18:252-256.

5 Al Aboud K, Al Aboud D: Multiple lesions of granuloma annulare on the hand in a patient with scabies. Clin Cosmet Investig Dermatol 2011;4:131-132.

-6 Walton SF, Pizzutto S, Slender A, et al: Increased allergic immune response to Sarcoptes scabiei antigens in crusted versus ordinary scabies. Clin Vaccine Immunol 2010;17:1428-1438. 


\section{Case Reports in

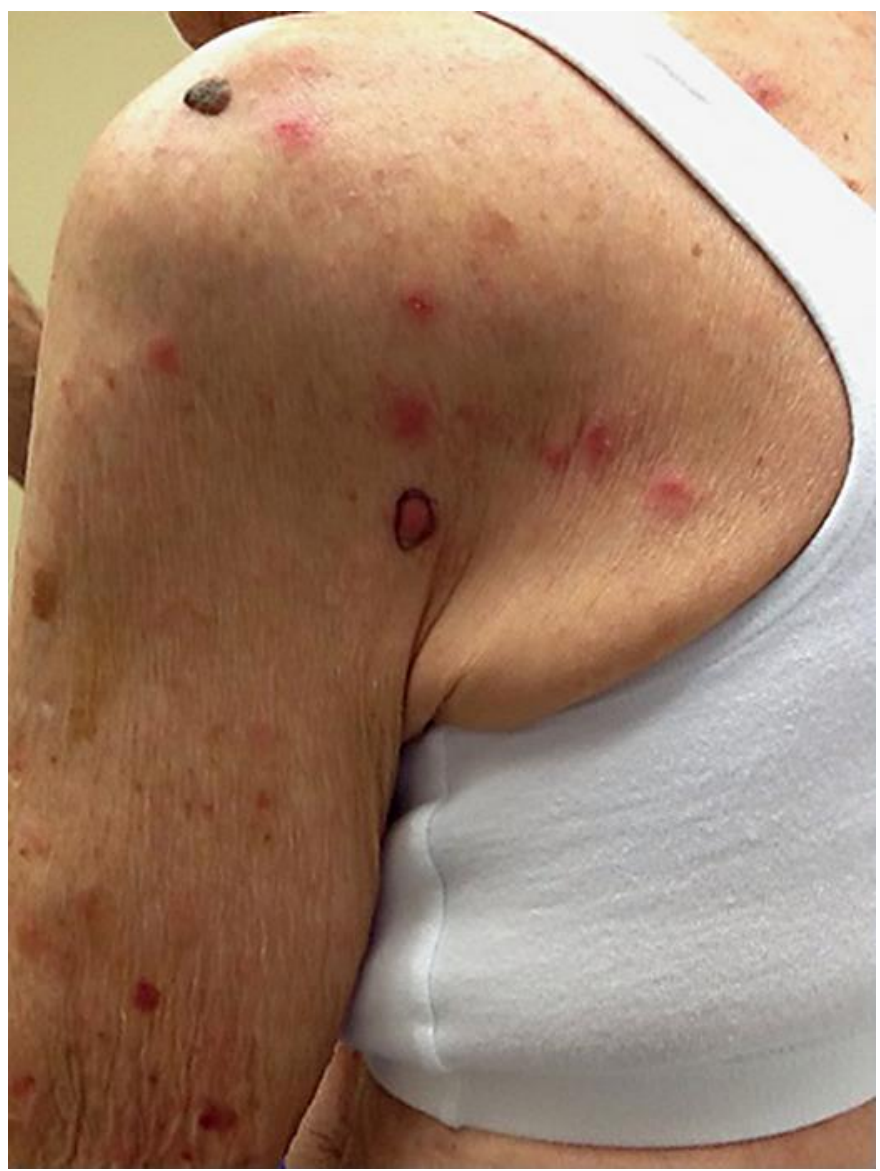

Fig. 1. Pink urticarial-like papules and plaques scattered over the trunk and extremities. 


\section{Case Reports in \\ Dermatology}

Case Rep Dermatol 2017;9:60-64

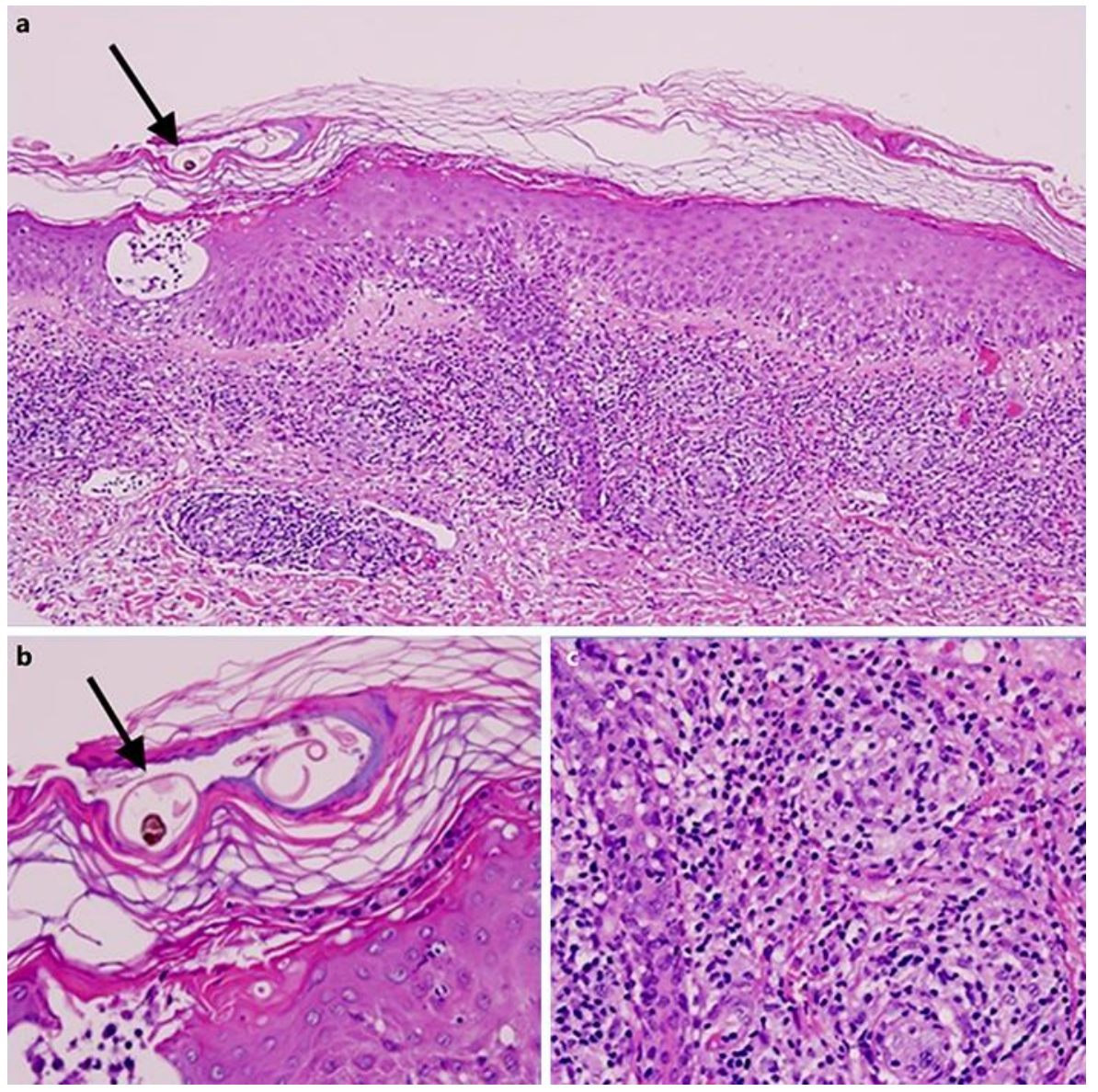

Fig. 2. a Granulomatous dermal dermatitis with intracorneal scabies mite. $\times 100$. b Scabies mite burrow. $\times 400$. c Non-necrotizing granulomas surrounded by lymphocytes and scattered eosinophils. $\times 400$. 